\title{
Use of Contourlets for Image Retrieval
}

\author{
Rajashekhar and Subhasis Chaudhuri \\ Department of Electrical Enginering, \\ IIT Bombay, Powai, India 400076 \\ \{raja, sc\}@ee.iitb.ac.in
}

\begin{abstract}
We propose a simple technique for content-based image retrieval (CBIR) using contourlets that possess not only the multi-resolution time frequency localization properties of wavelets but are also good at capturing the directional information. Statistical features extracted from contourlet coefficients on each subband of the decomposed image, capturing the textural properties, are combined with the color feature while doing the CBIR. The retrieval performance on a large image database shows that the proposed technique provides a better accuracy compared to other multi-resolution based approaches.
\end{abstract}

\section{Introduction}

Content-based image retrieval is now an actively pursued research area, and a comprehensive literature survey can be found in [1, 2. Although color is an effective feature, its use during retrieval may lead to some loss in discrimination power, if used alone. Texture is an important visual attribute whose properties are required for the identification of an object. Often one can efficiently encode the contents of many real world images, for example, cloud, bricks, building patterns, etc., in terms of textural characteristics. One such early work [3] is based on a statistical approach in which statistics derived from the cooccurence matrix are used to describe the texture. Another statistical scheme for wavelet-based texture retrieval scheme proposed in [4] is based on modeling the marginal distribution of the wavelet coefficients using a generalized Gaussian density (GGD) and computing the Kullback-Libler distance between the GGDs. Jhanwar et al. [5] have proposed a translation and illumination invariant retrieval scheme using motif cooccurence matrix. It uses an optimal Peano scan to locally encode the texture.

Normally the texture information is available at various scales. One natural method of texture analysis at various scales is to perform a multi-resolution analysis. Many researchers have explored the use of wavelets and Gabor filters for texture analysis at multi-resolution levels [6, 7, 8, 9]. Wavelets and Gabor filter approaches use separable bases during the image decomposition. However, the use of separable bases do not allow us to efficiently handle the presence of directional information hidden in the image. Indeed, this directional information is a very important cue in image content modeling since many natural images are not simply stacks of 1-D piecewise smooth scan-lines; discontinuity 
points (i.e. edges) are typically positioned along smooth curves along arbitrary directions owing to smooth boundaries of physical objects. Recently, Do et al. proposed in [10] the use of non separable bases in building contourlets for image decomposition which are very good in capturing the directional entities in the image. This inspired us to propose a simple technique of extracting the textural features using contourlets. We extract the statistical features such as mean and standard deviation on each subband of the decomposed Y component of the color image. We use these features to measure the similarity between two images. Subsequently we incorporate the color features. This method is simple and provides a favorably good retrieval accuracy compared to the existing texture retrieval methods. It may be noted that Do et al. 10. have also suggested the use of contourlet for image retrieval. However, their method requires the estimation of parameters for a hidden Markov model to parameterize the the contourlet tree structure. Thus it is computationally quite demanding and the performance depends on the fitness of the model. The proposed method is free from any model fitting.

\section{Content Modeling Using Contourlet Statistics}

The contourlet transform is an extension of the Cartesian wavelet transform in two dimensions using multiscale and directional filter banks. The contourlet expansion of an image consists of non separable bases oriented at various directions at multiple scales, with flexible aspect ratios. In addition to the multiscale time frequency localization properties of wavelets it offers a high degree of directionality (see more details in [10]). The Y component of the color image is decomposed into a lowpass subband and four, eight and sixteen bandpass directional subbands, respectively. Now we obtain 29 directional bandpass subbands at different scales for each image. Let $S_{m, n}$ be contourlet coefficients at the $m^{\text {th }}$ scale and $n^{\text {th }}$ directional subband. For example, for a 3-layer decomposition, the subbands are $S_{\mathbf{0 , 0}}, S_{\mathbf{1}, \mathbf{1}}, \cdots S_{\mathbf{1 , 4}}, S_{\mathbf{2}, \mathbf{1}} \cdots \cdots S_{\mathbf{3}, \mathbf{1 6}}$. We then compute the mean $\mu_{m, n}$ and standard deviation (STD) $\sigma_{m, n}$ features of the computed contourlet coefficients on each subband of the decomposed image using the following equations.

$$
\mu_{m, n}=\frac{\sum_{i, j}\left|S_{m, n}(i, j)\right|}{x_{m, n}}, \quad \sigma_{m, n}=\left[\frac{\sum_{i, j}\left|S_{m, n}(i, j)-\mu_{m, n}\right|^{2}}{x_{m, n}}\right]^{1 / 2},
$$

where $x_{m, n}$ is the number of components in the $(m, n)^{t h}$ subband. A feature vector is now constructed using $\mu_{m, n}$ and $\sigma_{m, n}$. For 3 levels of decomposition, the resulting contourlet feature vector $(\mathrm{CF})$ is given as $f=\left[\mu_{\mathbf{0}, \mathbf{0}}, \sigma_{\mathbf{0}, \mathbf{0}}, \mu_{\mathbf{1}, \mathbf{1}}, \sigma_{\mathbf{1}, \mathbf{1}} \ldots\right.$ $\left.\mu_{1,4}, \sigma_{1,4}, \mu_{2,1}, \sigma_{2,1} \cdots \mu_{2,8}, \sigma_{2,8} \mu_{3,1}, \sigma_{3,1} \cdots, \mu_{3,16}, \sigma_{3,16}\right]$.

\section{Textural Similarity}

Measuring the similarity among images is of central importance for retrieving images by content. After having computed the textural features we match the 
textural features of the query and database images using the Canberra distance metric [7]. We weigh the standard deviations on each subband differently while computing this distance metric. Standard deviations of subband images give a measure of amount of details in that subband. Since a texture mainly consists of quasi-periodic spatial variations, we expect the higher frequency subbands (lower levels of decomposition) to contain more texture information, and we give a higher weight to these subbands. Also if the image is noisy, only the lower bands are affected and the overall distance does not change drastically due to lower weights. We do not weigh the mean of the subbands since it provides an average behavior over the subbands.

We normalize the individual feature vector components before finding the distance between the two images. If $f_{q}$ and $f_{p}$ are the feature vectors of the query image and the database image, the Canberra distance $d_{c n t}$ [7] is given by

$$
\operatorname{Canb}\left(f_{p}, f_{q}\right)=d_{c n t}=\sum_{m, n} \frac{\left|\mu_{m, n}^{p}-\mu_{m, n}^{q}\right|+\omega_{m, n}\left|\sigma_{m, n}^{p}-\sigma_{m, n}^{q}\right|}{\left|\mu_{m, n}^{p}+\omega_{m, n} \sigma_{m, n}^{p}\right|+\left|\mu_{m, n}^{q}+\omega_{m, n} \sigma_{m, n}^{q}\right|},
$$

where $\omega_{m, n}=\frac{1}{2^{m-1}}$ and the superscript relates to a given image.

\section{Inclusion of Color Feature}

As discussed in section 2 we extract the contourlet features considering the $\mathrm{Y}$ component of the color image in YCbCr plane. We generalize the CBIR problem by incorporating the color information. The color histogram describes the global color distribution in an image [1]. We compute three separate histograms for $\mathrm{R}$, $\mathrm{G}$, and B planes of the color image. We use the Euclidean distance to compute the color similarity $\left(d_{c}\right)$ between the query and database images. Then the over all similarity $(d)$ is computed by combining both the similarities using appropriate weights.

$$
d=d_{c n t}+\beta d_{c}
$$

where $\beta$ the relative importance between the contourlet and color features. We select the weight $\beta$ in an adhoc basis so that both the components in the distance measure $d$ have nearly equal contributions.

\section{$5 \quad$ Experimental Results}

We tested the performance of the proposed method using various types of query images. The size of the database is about 5000, consisting of images of both natural and manmade objects. Each image in the database is decomposed upto 3 levels using the contourlet transform. In all the experimental results the image displayed first is the query image and ranking goes from left to right and top to bottom. 

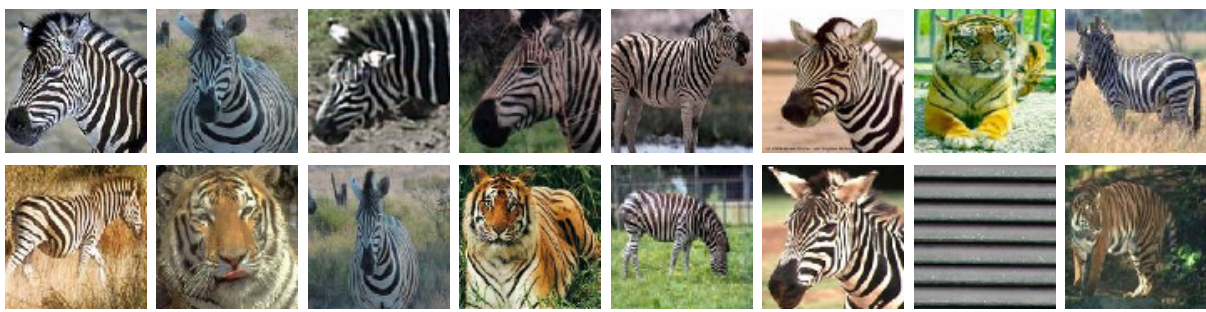

Fig. 1. Retrieved images using the contourlet features with the top left image as the query image. Here the contourlet features are computed up to the third level of decomposition.
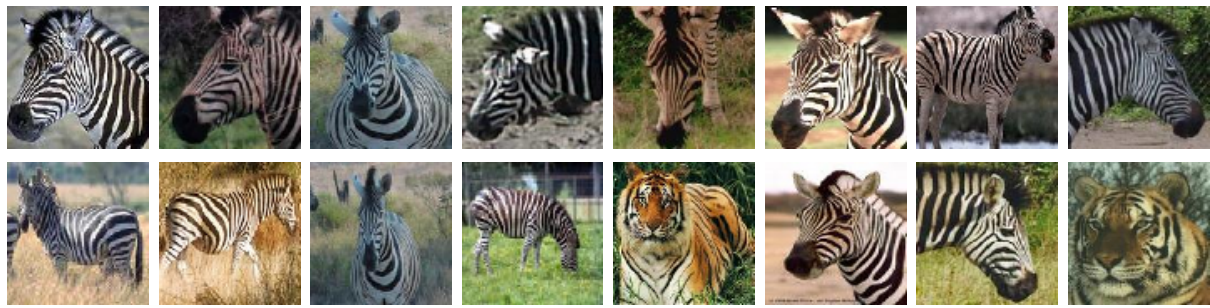

Fig. 2. Retrieved images using the weighted contourlet features (WCF) with the top left image as the query image

First we demonstrate the performance using contourlet features having equal weights ie, $\omega_{m, n}=1 \forall(m, n)$. Since contourlet features are good at capturing the geometrical structures such as smooth contours in natural images we retrieved a good number of relevant images. Since all the contourlet features are weighed uniformly, despite having top 5 relevant retrievals, we end up with a few false retrievals. The retrieved images using the zebra image as a query are shown in Figure 1. It may be noticed that although many retrieved images are relevant to the query a few images such as the images of the tiger and the horizontal texture do not correspond very well to the query image. We then consider the
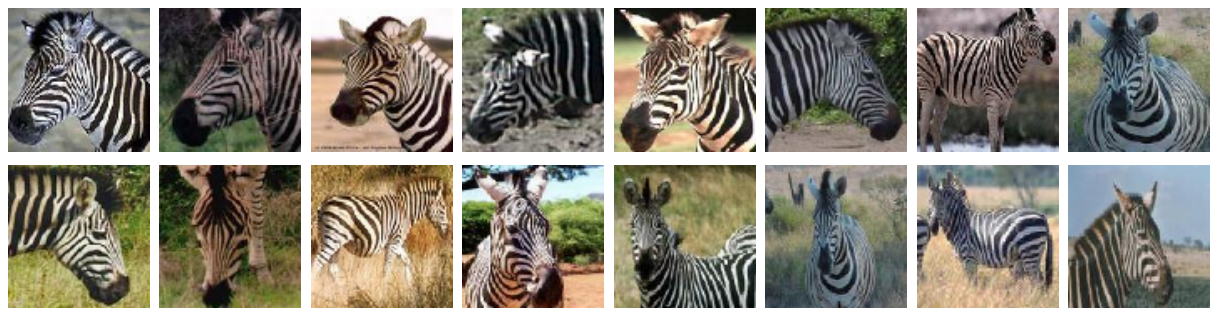

Fig. 3. Retrieved images using the combination of WCF and color features 

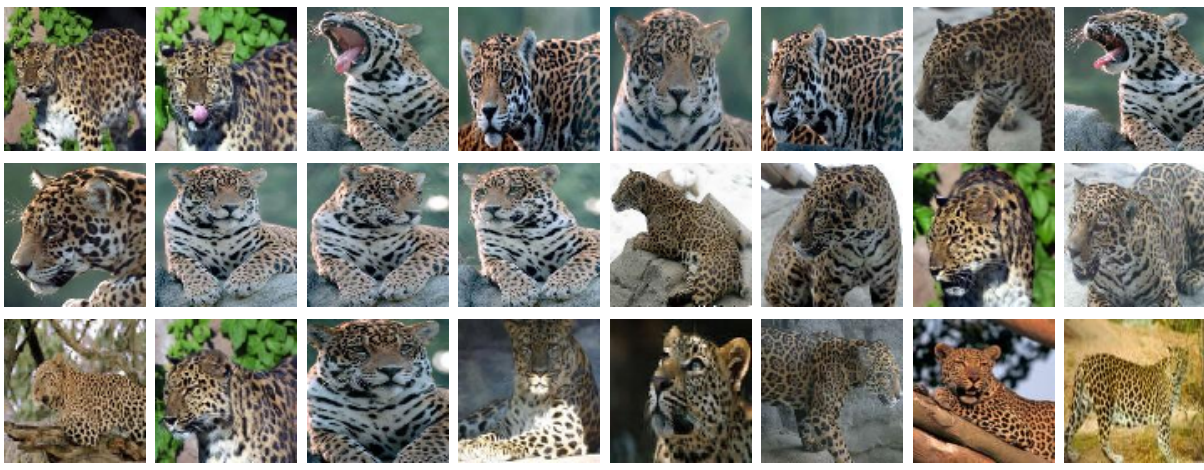

Fig. 4. Results of retrieval using both the WCF and color features for another query

use of the weighted contourlet features while doing CBIR. Figure 2 shows the corresponding retrieved images using the same zebra image as a query. In the top 15 retrieved images, shown here most of them correspond very well to the query image. Even then one may find a few irrelevant ones such as the tiger. This is due to the fact that the tiger has stripes similar to the zebra. We do note that a zebra and a tiger have different colors. We then combined the contourlet features with the color feature and repeated the CBIR experiment for the same image as the query. It may be noticed that in the retrieved list (see Figure 3), all the images correspond very well to the query image. This improves the retrieval accuracy further.

We then browsed the image database for similarity using a panther image as the query. The results in Figure 4 show the top 23 retrieved similar images. We compared the retrieval results of the proposed method with the results obtained using rotated wavelet filter (RWF), discrete wavelet filter (DWT) and RWF + DWT, and Gabor wavelets $[6$. We find that the retrieval results of the proposed one is superior to the above CBIR methods.

The proposed method is also computationally very efficient. The table in Figure 5 (b) provides a detailed comparison of average recall rates and retrieval time considering top 30 retrievals for combination of weighted contourlet feature (WCF) and color, contourlet feature (CF), DWT, RWF, combination of DWT and RWF, and Gabor wavelet method proposed by [6]. They have reported the Gabor wavelet-based texture image retrieval results using four scales and six orientations. For constructing the feature vector they have also used mean and standard deviation of the magnitude of the Gabor transform coefficients. The performance obtained with the standard DWT feature $(81 \%)$ is better than that obtained with RWF $(75.2 \%)$ features. The reason behind this is that RWF performs better for diagonally oriented texture images. The performance with combination of two feature sets (DWT + RWF) is better $(85.50 \%)$ than that using any of them alone. The performance obtained with the combination of WCF and color feature sets (94\%) is better than that using either WCF or CF as expected. In terms of retrieval time the existing Gabor wavelet based method 


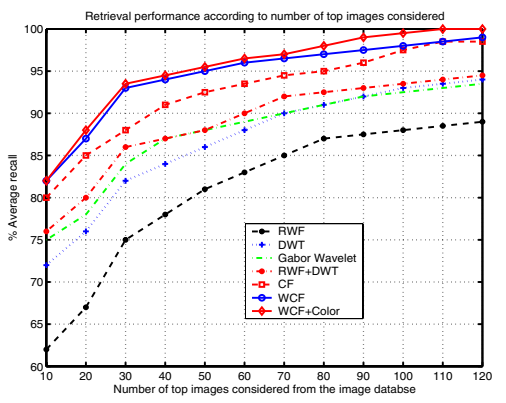

(a)

\begin{tabular}{|c|c|c|c|}
\hline & $\begin{array}{c}\text { Average recall } \\
\text { rate (\%) }\end{array}$ & $\begin{array}{c}\text { Retrieval time/per } \\
1000 \text { search }\end{array}$ & $\begin{array}{c}\text { Feature vector } \\
\text { length }\end{array}$ \\
\hline DWT & 81.00 & $0.28 \mathrm{sec}$ & 24 \\
\hline RWF & 75.20 & $0.21 \mathrm{sec}$ & 24 \\
\hline DWT + RWF & 85.50 & $0.47 \mathrm{sec}$ & 48 \\
\hline Gabor Wavelet & 84.52 & $1.9 \mathrm{sec}$ & 48 \\
\hline CF & 90.50 & $0.19 \mathrm{sec}$ & 58 \\
\hline WCF & 92.50 & $0.18 \mathrm{sec}$ & 58 \\
\hline WCF + Color & 94.00 & $0.22 \mathrm{sec}$ & $58+256$ \\
\hline
\end{tabular}

(b)

Fig. 5. (a) Comparison of recall rates of the proposed method with the methods based on RWF, DWT, DWT+RWF and Gabor Wavelet, respectively, (b) Average recall rates and retrieval times for some of the related CBIR methods and the proposed method Here WCF stands for weighted contourlet feature.

is the most expensive. The proposed retrieval system has been implemented using a combination of MATLAB and $\mathrm{C}$ on a Pentium IV, 2.2GHz machine. The retrieval time for the proposed method is quite close to the other wavelet based methods. It may be noted that the retrieval times for the first four methods given in table shown in Figure 5 (b) were on a P-III, $866 \mathrm{MHz}$ machine.

Figure 5(a) shows the recall rates for standard DWT, RWF, (DWT + RWF), Gabor wavelet, CF, and combination of WCF and Color based methods according to the number of top matches considered. From the figures it is clear that the retrieval performance of the combined WCF and Color feature set is superior to all the existing multi-resolution methods.

\section{Conclusion}

We have proposed the idea of using contourlet transform for CBIR. We have shown how the contourlet features are effective in capturing the directional information. The performance obtained with WCF is better than that of other multiresolution based CBIR schemes. We notice a further improvement in the retrieval accuracy with the inclusion of color feature. Computational requirement of the proposed method is very minimal. Thus the proposed method achieves both the important requirements such as high retrieval accuracy and less retrieval time for doing CBIR.

\section{References}

[1] Bimbo, A.D.: Visual Information Retrieval. Morgan Kaufman Publishers, Inc., San Francisco, USA, (2001)

[2] Rui, Y., Huang, T., Chang, S.F.: Image retrieval:current techniques promising directions and open issues. Journal of Visual Communication and Image Representation 10 (1999) 39-62 
[3] Haralick, R.M., Shanmugam, K., Dinstein, I.: Texture features for image classification. IEEE Trans.on System, Man and Cybernetics 3 (1973) 610-621

[4] Do, M.N., Vitterli, M.: Wavelet-based texture retrieval using generalized gaussian density and kullback-liebler distance. IEEE Transactions on Image Processing 11 (2002) 146-158

[5] Jhanwar, N., Chaudhuri, S., Seetharaman, G., Zavidovique, B.: Content based image retrieval using motif coocurence matrix". Image and Vision Computing 22 (2004) 1211-1220

[6] Manjunath, B.S., Ma, W.Y.: Texture features for browsing and retrieval of image data. IEEE Trans.on Pattern Analysis and Machine Inelligence 8 (1996) 837-841

[7] Kokare, M., Chatterji, B.N., Biswas, P.K.: Rotated wavelet based texture features for content based image retrieval. In: Fifth International Conference on Advances in Pattern Recognition. (2003) 243-247

[8] Smith, J., Chang, S.F.: Visual seek: A fully automated conent based query system. In: Proc. ACM Multimedia ACM Press, Newyork (1996) 87-98

[9] Simoncelli, E.P., Adelson, E.H.: Non-seperable extensions of quadrature mirror filters to multiple dimensions. In: Proc.of IEEE: Special issue on Multidimensional Signal Processing. Volume 78. (1990) 652-664

[10] Do, M.N., Vitterli, M.: The contourlet transform:an efficient directional multiresolution image representation. IEEE Transactions on Image Processing (to appear) (2004)

[11] Swain, M.J., Ballard, D.H.: Color indexing. International Journal of Computer Vision 7 (1991) 11-32 